\title{
A Challenging Case of Extraordinary - Long standing Forearm Non- union Treated with Extensive Debridement, Locking Plates, and Bone Grafting. Technical Considerations in Multiple-operated, Neglected Non- unions
}

\author{
George A Komnos ${ }^{1}$, Fotis Papageorgiou ${ }^{1}$, Konstantinos N Malizos ${ }^{1}$ \\ Learning Point of the Article:
}

The prompt use of locking plates and auto graft, respect and preservation of the local biology, and patient's compliance are of great importance when dealing with long-standing non-unions.

\section{Abstract}

Introduction: Forearm non-unions pose a significant treatment challenge to orthopedic surgeons. Repetitive treatment failures can lead to a devastating situation for the patient. Forearm function influences both elbow and wrist proper function.

Case Report: A functionless hand is presented, describing a longstanding non-union, treated with multiple surgeries before. A thorough debridement with respect to blood supply and local biology are of major importance before applying the locking plates along with the use of bone-graft. The reconstruction of the forearm converted a functionless arm to a fully functioning arm and the patient returned eventually to her previous activities.

Conclusion: Fixation with locking plates combined with the use of autograft can lead to very satisfactory results even in extraordinary cases, especially when attention is paid to local anatomy and blood supply.

Keywords: Forearm nonunion, locking plates, multiple surgeries.

\section{Introduction}

Forearm is an anatomical structure with unique interosseous space allowing the rotation of the radius around the ulna in prosupination. Due to this function forearm fractures must be treated as articular fractures and not as diaphyseal ones. Treatment of forearm fractures with compression plating presents a union rate of more than $95 \%[1,2]$. Factors affecting the result of forearm fixation apart from the surgical technique are osteoporosis, mechanism of injury, and patient's compliance [3]. Notable complications include nonunion, malunion, and refracture after implant removal [4]. Malunion and nonunion are relatively frequent, due to rotating forces from the supinator and pronator muscles [3]. In terms of nonunion management, pre-operative deformity, chronicity, presence of infection, bone loss, quality of the bone, and the soft-tissue envelope are variables that should be taken into consideration before choosing the appropriate method.

\section{Case Report}

A35-year-old woman was referred to the outpatient's clinic with a long-standing nonunion, persistent pain on a functionless hand and wrist, and a severe angular deformity of her left forearm. Eight years ago, the patient fell from the horse back and sustained a closed bifocal fracture of the radius (radial shaft and distal radius) and a fracture of the ulnar shaft (Fig. 1), initially treated with open reduction and internal fixation (plates and screws for the forearm diaphyseal fractures and a palmar plate for the distal radius fracture) (Fig. 2). According to past medical

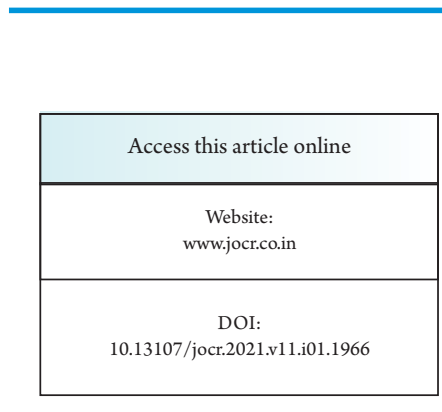

Author's Photo Gallery

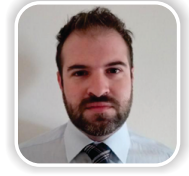

Dr. Fotis Papageorgiou

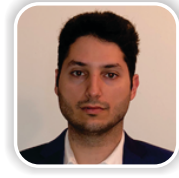

Dr. George A Komno

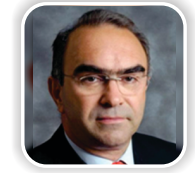

Dr. Konstantinos N Malizos

${ }^{1}$ Department of Orthopedics, General University Hospital of Larisa, University of Thessaly, Larisa, Greece.

Address of Correspondence:

Dr. George A Komnos,

Department of Orthopedics, University of Thessaly, Larisa, 41110, Greece.

E-mail: gekomnos@hotmail.com

Journal of Orthopaedic Case Reports | pISSN 2250-0685 | eISSN 2321-3817 | Available on www.jocr.co.in | doi:10.13107/jocr.2021.v11.i01.1966 This is an Open Access article distributed under the terms of the Creative Commons Attribution Non-Commercial License (http://creativecommons.org/licenses/by-nc/3.0) which permits unrestricted non-commercial use, distribution, and reproduction in any medium, provided the original work is properly cited. 


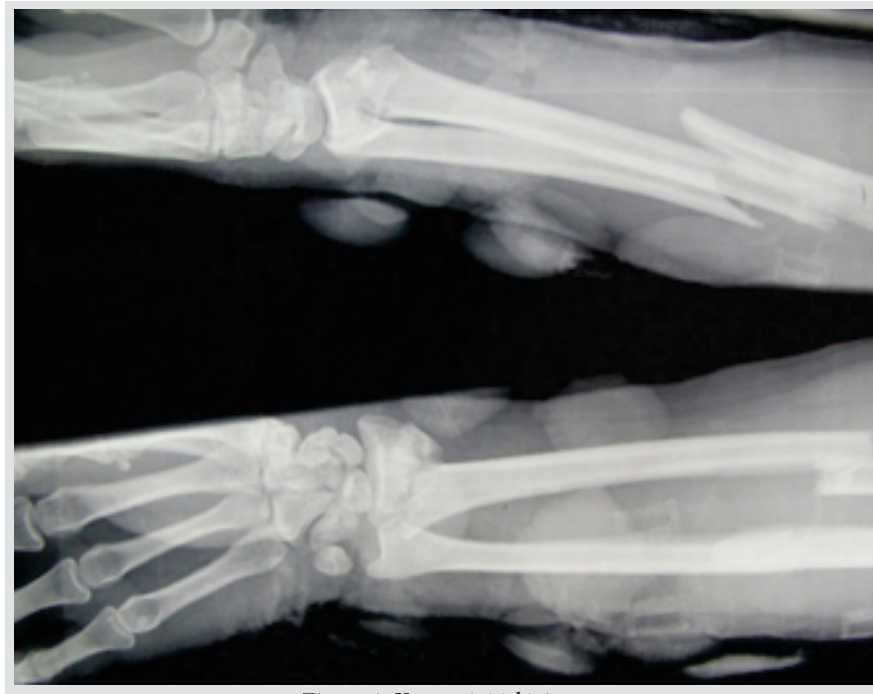

Figure 1: X-ray at initial injury.

records, 3 months after the initial procedure, she presented with persistent forearm pain and radiological signs of hardware failure and loosening. She underwent surgical debridement and revision of the osteosynthesis applying new plates and screws enhanced by bone grafting. Intraoperative sample cultures were obtained and one of them proved positive for Escherichia coli. The patient was subsequently given intravenous antibiotics for 3 weeks.

One year later, due to nonunion and presence of a bone gap, she underwent reoperation with "enhancement of the union potential" use of eptotermin alfa, bone morphogenetic proteins, and autologous iliac bone grafting, which were proved effective for the radial shaft fracture. Five months later, the ulnar nonunion and bone gap were treated with distraction osteogenesis with the use of an external fixator. Enhancements with two more surgical interventions with corticocancellous bone grafting were done with 4 months interval. The external fixation remained for 3 weeks after the last procedure, until clinical and radiological signs of union. The patient experienced a period of 4 years free of symptoms with no significant functional impairment. However, eventually she gradually established pain, swelling and severe deformity of her left forearm with magnetic resonance imaging and $\mathrm{Ga} 67$ bone scan raising suspicion for infection. She was admitted for surgical debridement, hardware removal, and intravenous and then oral

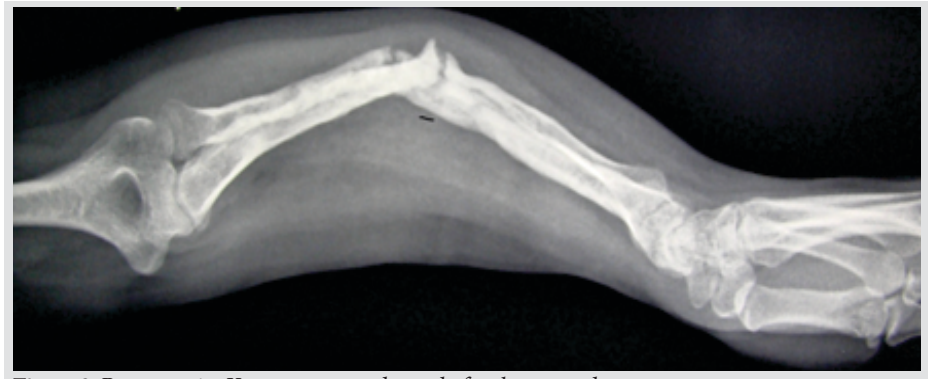

Figure 3: Pre-operative X-ray as presented to us, before last procedure.

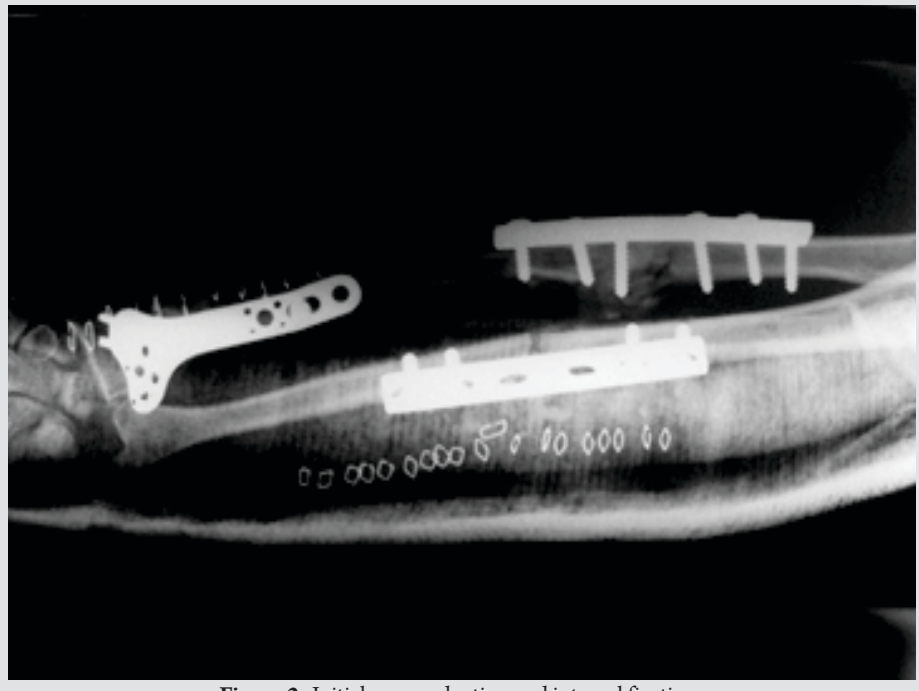

Figure 2: Initial open reduction and internal fixation

antibiotic treatment (ciprofloxacin and rifampicin) while intraoperative culture samples were negative. The patient's medical and family history is clear, while she referred smoking from her social history.

At her presentation in our department, she had a significant deformity with angulation of the forearm, lack of motion with pronation and supination $<10^{\circ}$, and severe functional loss, obliged to use a splint to use her hand. Elbow flexion was restricted to $20^{\circ}$ and wrist range of motion was $20-0-20^{\circ}$ (Fig. 3). Lack of swelling, erythema, and increased local temperature, or any other clinical sign of infection was detected. Laboratory tests were negative for infection (white blood cell: 6800, Creactive protein: 0.2 , and erythrocyte sedimentation rate: 7 $\mathrm{mm})$.

At the operating theater, after an extensive and demanding approach at the site of the non-union arduous approach of the nonunion, debridement was done along with excision of all non-viable bone segments, and shortening of about $2.5 \mathrm{~cm}$ so as to allow reduction without excessive stretching of the nerves and vessels. Internal fixation with reconstruction plates was carried out, augmented with posterior iliac crest autograft. Intraoperative cultures of soft tissues and bone were obtained to rule out infection (Fig. 4). The patient had a short hospital stay, the culture results were negative and she was discharged wearing a long arm splint for 6 weeks. The patient was followed

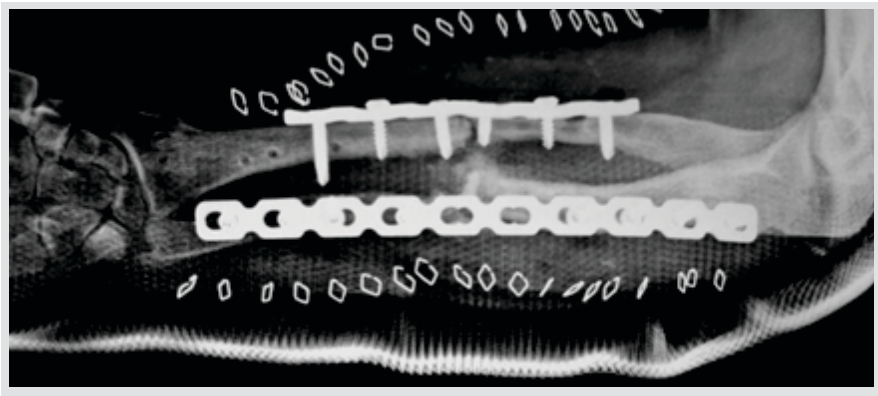

Figure 4: Post-operative X-ray. 

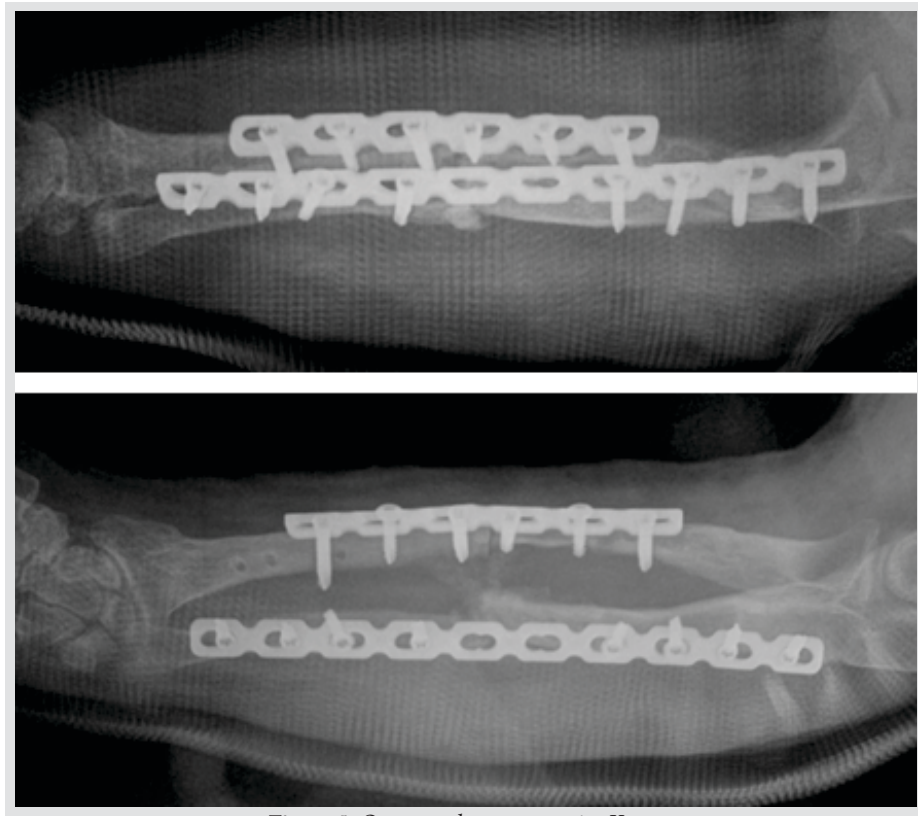

Figure 5: One-month post-operative X-ray

up at 1, 2, 4, and 7 months and 1 year postoperatively with clinical evaluation and radiological examination (Fig. 5, 6). At last follow-up supination and pronation of the forearm were approximately $100^{\circ}$. Elbow flexion was significantly improved at more than $90^{\circ}$ and range of motion of the wrist was 50-0-50.

\section{Discussion}

Nonunion of forearm fractures is a really challenging complication for both orthopedic surgeons and the patient themselves. In forearm fractures, a reliable, anatomical reduction is usually achieved using plates [5]. Success rates can reach or exceed $90 \%[2,6,7,8]$, while other operative techniques such as i.m. nailing seem to have lower union rates [9]. The main goal of operating treatment is to restore bone length, alignment, rotation, the three-dimensional shapes and relationships of radius and ulna $[10,11]$ and achieve the best result regarding pronation, supination, and grip strength. It is important to underline that forearm proper function also affects elbow and wrist function. Fixation stability and preservation of biologic environment are of major importance when treating these fractures. A cadaveric study has demonstrated that malunion of radius and ulna with angulation more than $20^{\circ}$ limits forearm rotation $[4,12]$. Our patient came to our department with almost $40^{\circ}$ of malalignment present.

Some of the operative indications for nonunion and malunion include severe pain, deformity, radio-ulnar joint instability, functional limitations, and restricted forearm range of motion $[13,14]$, which were all present in our patient. Factors related to fracture (comminution, open fracture, location of the fracture, and state of soft tissue), patient (age, smoking, and additional diseases), and initial treatment (implant used and surgical

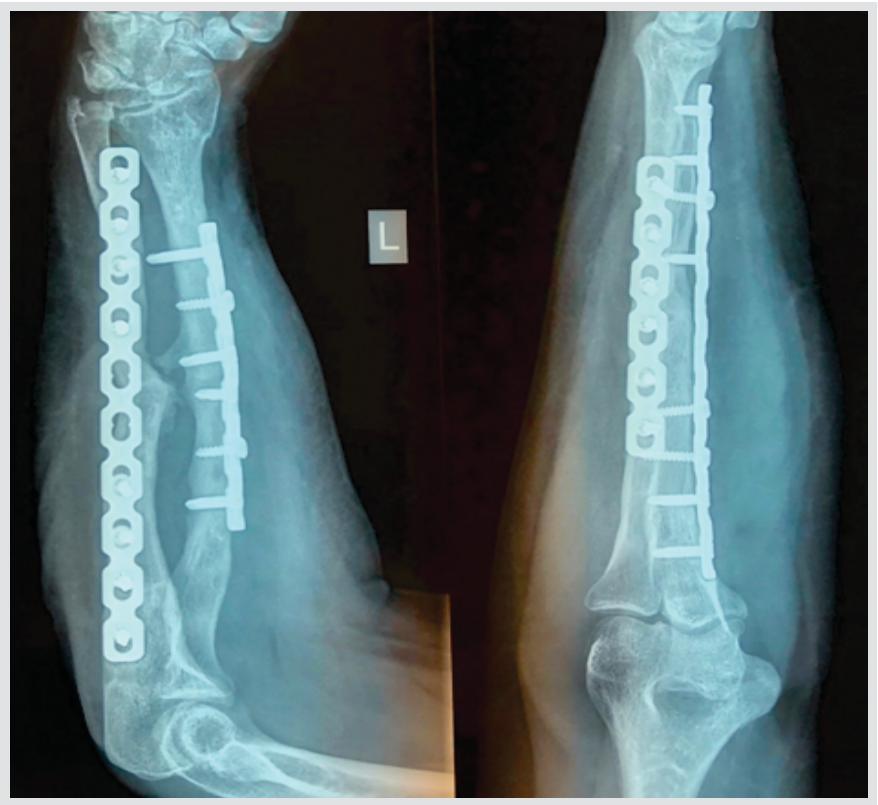

Figure 6: One-year post-operative $\mathrm{X}$-rays

technique) have been held responsible for forearm non-union $[3,14,15]$. During surgery, essential steps that have to be followed, which were all done during our operation, are removal of failed implant with simultaneous debridement of devitalized tissue, respect to blood supply and fixation with proper alignment and rotation $[5,15]$. Use of autografts in the treatment of nonunion of the long bones is still a matter of debate. We used posterior iliac crest autograft to enhance union as a large surgical debridement was done and the bone quality was compromised from the several procedures that took place before. Some of the disadvantages of plate fixation are the extensive dissection required, the risk of injury to neurovascular structures and the potential for synostosis [9]. Fortunately, we made no damage in neurovascular structure, while a very small amount of synostosis is present at 1-year follow-up, without restricting patient's every day activity.

Another important issue in these cases is that every nonunion should also be investigated for infection $[4,15]$. Although in our case there were no signs of infection before the surgery, we did obtain cultures from the operative field, which finally proved negative. One of the facts that we had also to deal with during reconstruction was that due to extensive debridement to discard non-viable bone, we had a final shortening of about 2.5 $\mathrm{cm}$ in bone structures. The high number of the operations undertaken before was one of the reasons for the big amount of non-viable bone segment, probably due to bad blood supply. At her last follow-up visit, we measured the patient's forearms and we estimated that the length difference between her forearms was approximately $8 \%$. Difference in upper limb length leads to less severe dysfunction than this in lower limb. Therefore, indications for upper limb lengthening are relatively rare. Despite this small bone inequality, the patient referred no 
problem or difficulty in her everyday life deriving from it. The splint was applied for 6 weeks due to the patient's history and compliance [3].

\section{Conclusion}

It seems that the nonunion in this patient developed due to the previous insufficient fixation and the insufficient biology in the fracture side. Furthermore, patient's compliance played also a role since she did not quit smoking during recovery. We suggest that assiduous debridement with respect to local biology and the fixation with locking plates combined with the use of Clinical Message

Extraordinary and neglected nonunions that lead to a functionless hand can be treated with the prompt use of locking plates and autograft iflocal biology is preserved.

\section{References}

1. Kamrani RS, Farhoud AR, Nabian MH, Farhadi L. Treatment of nonunion of forearm bones using radial forearm bone flap. Trauma Mon 2015;20:e22622.

2. Chapman MW, Gordon JE, Zissimos AG. Compression-plate fixation of acute fractures of the diaphyses of the radius and ulna. J Bone Joint Surg Am 1989;71:159-69.

3. Kuyucu E, Koçyiğit F, Ciftçi L. The importance of patient compliance in nonunion of forearm fracture. Int J Surg Case Rep 2014;5:598-600.

4. Schulte LM, Meals CG, Neviaser RJ. Management of adult diaphyseal both-bone forearm fractures. J Am Acad Orthop Surg 2014;22:437-46.

5. Lapcin O, Arıkan Y, Yavuz U, Akman YE, Çetinkaya E, Gür V, et al. Evaluation of outcomes in aseptic non-unions of the forearm bones in adults treated with LCP and autograft. Ulus Travma Acil Cerrahi Derg 2016;22:283-9.

6. Anderson LD, Sisk D, Tooms RE, Park WI 3rd. Compressionplate fixation in acute diaphyseal fractures of the radius and ulna.J Bone Joint Surg Am 1975;57:287-97.

7. Hertel R, Pisan M, Lambert S, Ballmer FT. Plate osteosynthesis of diaphyseal fractures of the radius and ulna. Injury 1996;27:545-8.

8. Ross ER, Gourevitch D, Hastings GW, Wynn-Jones CE, Ali S. Retrospective analysis of plate fixation of diaphyseal fractures of the forearm bones. Injury 1989;20:211-4.

9. Richard MJ, Ruth DS, Aldridge JM 3rd. Malunions and nonunions of the forearm. Hand Clin 2007;23:235-43.

10. Faldini C, Pagkrati S, Nanni M, Menachem S, Giannini S. Aseptic forearm nonunions treated by plate and opposite fibular autograft strut. Clin Orthop Relat Res 2009;467:2125-34.

11. Moroni A, Caja VL, Sabato C, Rollo G, Zinghi G. Composite bone grafting and plate fixation for the treatment of nonunions of the forearm with segmental bone loss: A report of eight cases.J Orthop Trauma 1995;9:419-26.

12. Matthews LS, Kaufer H, Garver DF, Sonstegard DA. The effect on supination-pronation of angular malalignment of fractures of both bones of the forearm. J Bone Joint Surg Am 1982;64:14-7.

13. Borrelli J Jr., Prickett WD, Ricci WM. Treatment of nonunions and osseous defects with bone graft and calcium sulfate. Clin Orthop Relat Res 2003;411:245-54.

14. Jayakumar P, Jupiter JB. Reconstruction of malunited diaphyseal fractures of the forearm. Hand (N Y) 2014;9:265-73.

15. Parihar M, Ahuja D. Infected nonunion of radius and ulnastrategy of approach.J Orthop Case Rep 2012;2:26-31.

\section{Source of Support: Nil}

Consent: The authors confirm that informed consent was obtained from the patient for publication of this case report

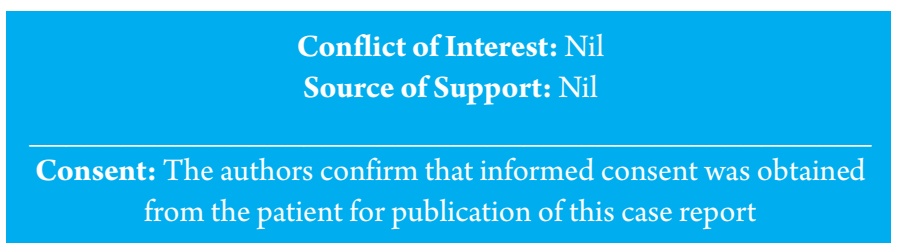

\section{How to Cite this Article}

Komnos GA, Papageorgiou F, Malizos KN. A Challenging Case of Extraordinary - Long standing Forearm Non-union Treated with Extensive Debridement, Locking Plates, and Bone Grafting. Technical Considerations in Multiple-operated, Neglected Non-unions. Journal of Orthopaedic Case Reports 2021 January; 11(1):63-66 